\title{
SPANS AND INTERSECTIONS OF ESSENTIALLY REDUCING SUBSPACES
}

\author{
MICHAEL J. HOFFMAN
}

\begin{abstract}
If $P$ and $Q$ are the projections onto essentially reducing subspaces $M$ and $N$ for an operator, the closed linear span and the intersection of $M$ and $N$ need not be essentially reducing or even essentially invariant. However, they are if $M+N$ is closed, in particular if $P Q=Q P$ or if $P Q$ is compact.
\end{abstract}

1. Introduction. If $P$ is the orthogonal projection of separable Hilbert space $H$ onto a closed subspace $M$, then $M$ is invariant under a bounded linear operator $T$ when $T P-P T P=0$. It reduces $T$ when $T P-P T=0$. This paper examines these notions modulo the compact operators. Let $L(H)$ be the algebra of all bounded linear operators on $H$, and $K(H)$ be the ideal of compact operators.

Definition. If $S \subseteq L(H)$ and $P$ is the orthogonal projection onto a closed subspace $M$, then $M$ (or $P$ ) is essentially invariant for $S$ if $T P-P T P$ is in $K(H)$ for every $T$ in $S$. It is essentially reducing if $T P-P T$ is in $K(H)$ for every $T$ in $S$.

In contrast to the situation for invariant subspaces, every operator is known to have nontrivial (i.e. infinite dimension and codimension) essentially invariant and reducing subspaces. Fillmore, Stampfli, and Williams show that if $c$ is in the boundary of the essential spectrum of $T$, there is a projection $P$ of infinite rank and nullity such that $(T-c) P$ is compact, so $(1-P) T P \in$ $K(H)[4$, p. 815]. Recent results of Voiculescu show that separable algebras of operators have nontrivial essentially invariant projections [1, p. 344], [7]. In particular, the $C^{*}$-algebra generated by $T$ and 1 does, so $T$ has essentially reducing subspaces.

The examples in $\$ 2$ show that much of the lattice structure of the sets of invariant and reducing subspaces for an operator fails for these weaker notions. We do have the following result.

Proposition 1.1. Suppose $M$ and $N$ are closed subspaces of Hilbert space $H$ and that $M+N$ is closed. If $M$ and $N$ are essentially invariant (reducing) for an operator $T$, then $M+N$ and $M \cap N$ are also.

This certainly applies when $M$ and $N$ are orthogonal. It also applies if they

Received by the editors December 3, 1977 and, in revised form, March 23, 1978.

AMS (MOS) subject classifications (1970). Primary 47A15, 47A55.

Key words and phrases. Essentially invariant subspaces, essentially reducing subspaces, compact perturbations, Calkin algebra.

(1) American Mathematical Society 1978 
are almost orthogonal in the sense that the product of the corresponding projections is compact.

Proposition 1.2. If $P$ and $Q$ are projections onto closed subspaces $M$ and $N$ and $P Q$ is compact, then $M+N$ is closed.

All projections in this paper are assumed to be selfadjoint. Propositions 1.1 and 1.2 will be proved in $\S 4$. Proposition 1.1 also applies if the corresponding projections $P$ and $Q$ commute. In that case, $P Q$ is the projection onto $M \cap N$, and $P+Q-P Q$ is that onto $M+N$. The latter is closed since it is the range of a continuous projection. So the closed linear span $M \vee N$ is $M+N$; Proposition 1.1 applies; and the closed linear span and intersection are essentially invariant if $M$ and $N$ are.

Corollary 1.3. If $P$ and $Q$ are projections onto subspaces $M$ and $N$ and if $P Q=Q P$, then $M \cap N$ and $M+N$ are essentially invariant (reducing) whenever $M$ and $N$ are.

This can also be shown directly. The invariant case holds because

$$
T P Q-P Q T P Q=Q(T P-P T P) Q+(T Q-Q T Q) P
$$

and

$$
\begin{aligned}
T(P+Q-P Q) & -(P+Q-P Q) T(P+Q-P Q) \\
& =(1-P)(T Q-Q T Q)+(1-Q)(T P-P T P)(1-Q) .
\end{aligned}
$$

The reducing case follows from

$$
T P Q-P Q T=(T P-P T) Q+P(T Q-Q T)
$$

and

$$
\begin{aligned}
T(P+Q-P Q)-(P+ & Q-P Q) T \\
& =(T P-P T)(1-Q)+(1-P)(T Q-Q T)
\end{aligned}
$$

Before presenting a few examples, we list some immediate consequences of the definitions.

Proposition 1.4. (i) $M$ is essentially invariant for $T$ if and only if $M^{\perp}$ is essentially invariant for $T^{*}$.

(ii) $M$ is essentially reducing if and only if $M^{\perp}$ is essentially reducing if and only if both $M$ and $M^{\perp}$ are essentially invariant.

(iii) $M$ is essentially reducing if it has finite dimension or codimension in $H$.

(iv) If $S \subseteq L(H)$ is such that $T^{*}$ is in $S$ whenever $T$ is in $S$, then any subspace essentially invariant for $S$ essentially reduces $S$.

2. Counterexamples. The intersection of a family of essentially reducing subspaces need not be essentially invariant. Also, their span need not be, even if the family is pairwise orthogonal. An easy way to see this is to notice that any subspace is an intersection of spaces of finite codimension and is the closed linear span of one dimensional spaces. 
EXAmple 2.1. Let $T$ be the unilateral shift on an orthonormal basis $\left(e_{1}, e_{2}, e_{3}, \ldots\right)$ for $H$. That is, $T e_{n}=e_{n+1}$. Let $X_{n}$ be the space spanned by $e_{n}$, and let $Y_{n}=X_{n}{ }^{\perp}$. Let $M=Y_{1} \cap Y_{3} \cap Y_{5} \cap \cdots=$ the closed linear span of $\left(X_{2}, X_{4}, X_{6}, \ldots\right)$. Then the $X_{n}$ and $Y_{n}$ are all essentially reducing since they have finite dimension and codimension respectively. But $M$ is not essentially invariant. If $P$ is the projection of $H$ onto $M$, then $(T P-P T P) e_{n}$ $=0$ for odd $n$ and is $e_{n+1}$ for even $n$. So TP - PTP is not compact. There can be no smallest essentially invariant subspace containing all the $X_{2 n}$ since it would be contained in $Y_{1} \cap Y_{3} \cap \cdots$ and so would have to be $M$.

We saw above that the span and intersection of two essentially invariant subspaces are also essentially invariant if the corresponding projections commute. To seek weaker sufficient conditions, we might consider the canonical projection

$$
\pi: L(H) \rightarrow L(H) / K(H)
$$

onto the Calkin algebra. By analogy to 1.3 we could assume $\pi(P Q-Q P)=$ 0 , that is, $P Q-Q P \in K(H)$. Then $\pi(P Q)$ and $\pi(P+Q-P Q)$ would be selfadjoint idempotents in $L(H) / K(H)$, so a result of Calkin [2, p. 850 , Theorem 24] shows there are projections in the corresponding preimages. Since they are compact perturbations of $P Q$ and $P+Q-P Q$, algebra like that following 1.3 above shows that these projections are essentially invariant or reducing if $P$ and $Q$ are. Unfortunately they need not have much to do with $P H \cap Q H$ and $P H \vee Q H$. Indeed, $Q$ could be a compact perturbation of $P$ and the result would fail spectacularly as the following examples show.

EXAMPLE 2.2. For $0 \leqslant d \leqslant 1$, the matrix

$$
Q_{d}=\left[\begin{array}{ccc}
1-d & i\left(d-d^{2}\right)^{1 / 2} & 0 \\
-i\left(d-d^{2}\right)^{1 / 2} & d & 0 \\
0 & 0 & 0
\end{array}\right]
$$

defines a rank one projection on $\mathbf{C}^{3}$. Let $A$ be the matrix

$$
A=\left[\begin{array}{lll}
0 & 0 & 0 \\
0 & 0 & 0 \\
0 & 1 & 0
\end{array}\right]
$$

and define operators on $H=\mathbf{C}^{3} \oplus \mathbf{C}^{3} \oplus \mathbf{C}^{3} \oplus \ldots$ by

$$
\begin{aligned}
& P=Q_{0} \oplus Q_{0} \oplus Q_{0} \oplus \ldots \\
& Q=Q_{1 / 2} \oplus Q_{1 / 3} \oplus Q_{1 / 4} \oplus \ldots \text { and } \\
& T=A \oplus A \oplus A \oplus \ldots
\end{aligned}
$$

Then $T P-P T=0$, so $P H$ reduces $T$. Also,

$$
T Q-Q T=\bigoplus_{n=2}^{\infty}(1 / n)\left[\begin{array}{ccc}
0 & 0 & 0 \\
0 & 0 & 0 \\
-i(n-1)^{1 / 2} & 1 & 0
\end{array}\right]
$$


which is compact. So $Q H$ essentially reduces $T$. Most of $P$ and $Q$ are quite close together so that

$$
P Q-Q P=\bigoplus_{n=2}^{\infty} i(n-1)^{1 / 2} / n\left[\begin{array}{lll}
0 & 1 & 0 \\
1 & 0 & 0 \\
0 & 0 & 0
\end{array}\right]
$$

which is compact. In fact, $P-Q$ is compact. Nevertheless, the projection on the closed linear span, $P H \vee Q H$, is

$$
R=\left[\begin{array}{lll}
1 & 0 & 0 \\
0 & 1 & 0 \\
0 & 0 & 0
\end{array}\right] \oplus\left[\begin{array}{lll}
1 & 0 & 0 \\
0 & 1 & 0 \\
0 & 0 & 0
\end{array}\right] \oplus \ldots
$$

so that $T R-R T=T R-R T R=T$ which is not compact. So $R$ is not essentially invariant for $T$ much less reducing.

EXAMPLE 2.3. To obtain an example for intersections, we look at the orthogonal complements, $(1-P) H$ and $(1-Q) H$, of the spaces of Example 2.2. These essentially reduce $T$. The projections $1-P$ and $1-Q$ have compact commutator. But the projection on the intersection is $S=1-R$, and $T S-S T=R T-T R=-T$, so $S$ does not essentially reduce $T$. It is essentially invariant since $T S-S T S=0$, but it is not essentially invariant for $T^{*}$ since $T^{*} S-S T^{*} S=T^{*}$, although $1-P$ and $1-Q$ do essentially reduce $T^{*}$.

It is thus not sufficient to assume a compact commutator for $P$ and $Q$. Proposition 1.1 suggests that the problem is that $M+N$ is not closed. Proposition 1.2 says that if we make the stronger assumption that $P Q$ is compact, we do get the desired result.

3. Technical lemmas. The angle between subspaces. The basic fact which makes Propositions 1.1 and 1.2 work is that the sum of two subspaces with trivial intersection is closed if and only if there is a positive angle between them. This is contained in the following lemma whose content is reasonably well known. See for example Proposition 1.1 on page 21 of [3] and the material following it. A proof is included here for completeness and to bring the computational portions of the lemma together.

Lemma 3.1. Let $P$ and $Q$ be projections onto closed subspaces $M$ and $N$. Let $U=(M \cap N)^{\perp} ; V=M \ominus(M \cap N) ;$ and $W=N \ominus(M \cap N)$. Then the following are equivalent.

(i) $M+N=(V+W) \oplus(M \cap N)$ is closed.

(ii) $V+W$ is closed.

(iii) There is a constant $c$ with $0 \leqslant c \lesseqgtr 1$ such that $|\langle v, w\rangle| \leqslant c\|v\|\|w\|$ for every $v \in V$ and $w \in W$.

With the same constant $c$, these are also equivalent to the following estimates.

(iv) $(1-c)\left(\|v\|^{2}+\|w\|^{2}\right) \leqslant\|v+w\|^{2} \leqslant(1+c)\left(\|v\|^{2}+\|w\|^{2}\right)$ for every $v \in V$ and $w \in W$. 
(v) $\left\|\left.P Q\right|_{U}\right\| \leqslant c$.

(vi) $\left\|\left.Q P\right|_{U}\right\| \leqslant c$.

(vii) $\left\|\left.P\right|_{W}\right\| \leqslant c$.

(viii) $\left\|\left.Q\right|_{V}\right\| \leqslant c$.

Proof. If $f \in M ; g \in N$; and $S$ is the projection onto $M \cap N$, then

$$
f+g=(1-S) f+(1-S) g+S(f+g) \in V+W+(M \cap N) .
$$

Since $V+W \perp M \cap N$, we have $(V+W) \oplus(M \cap N)=M+N$. Since $M \cap N$ is closed, (ii) implies (i). For the converse, suppose $M+N$ is closed, and let $\left(f_{n}\right)$ be a Cauchy sequence in $V+W$. Then $f_{n} \rightarrow f$ for some $f$ in $M+N$. Let $f=u+g$ with $u$ in $V+W$ and $g$ in $M \cap N$. Then $\left\|f_{n}-f\right\|^{2}=$ $\left\|f_{n}-u\right\|^{2}+\|g\|^{2}$. So $g=0$ and $f=u$ which is in $V+W$. Thus $V+W$ is closed, and (i) implies (ii).

Now let $V+W$ be closed and consider a Banach space direct sum $B$ of $V$ and $W$ with norm given by

$$
\|(v, w)\|=\|v\|+\|w\| .
$$

The map $(v, w) \mapsto v+w$ of $B$ onto $V+W$ is one-to-one since $V \cap W=(0)$. It is continuous by the triangle inequality. By the open mapping theorem, there is a constant $d$ such that $\|v\|+\|w\| \leqslant d\|v+w\|$ for all $v$ in $V$ and $w$ in $W$. So

$$
\|v\|^{2}+2\|v\|\|w\|+\|w\|^{2} \leqslant d^{2}\|v+w\|^{2}=d^{2}\left(\|v\|^{2}+\|w\|^{2}+2 \operatorname{Re}\langle v, w\rangle\right) .
$$

Thus $2 / d^{2} \leqslant 1+\operatorname{Re}\langle v, w\rangle$ for all unit vectors $v$ and $w$. We may multiply by a complex number of magnitude one to assume $\operatorname{Re}\langle\boldsymbol{v}, w\rangle \leqslant 0$. So $2 / d^{2} \leqslant 1-|\langle v, w\rangle|$ for all unit vectors. For arbitrary $v$ in $V$ and $w$ in $W$ we have $|\langle v, w\rangle| \leqslant\left(1-2 / d^{2}\right)\|v\|\|w\|$. So (ii) implies (iii).

Since $\|v+w\|^{2}=\|v\|^{2}+\|w\|^{2}+2 \operatorname{Re}\langle v, w\rangle$, assumption of (iii) gives

$$
\|v\|^{2}+\|w\|^{2}-2 c\|v\|\|w\| \leqslant\|v+w\|^{2} \leqslant\|v\|^{2}+\|w\|^{2}+2 c\|v\|\|w\| .
$$

But this gives assertion (iv) since $2\|v\|\|w\| \leqslant\|v\|^{2}+\|w\|^{2}$.

Now suppose $\left(f_{n}\right)$ is a Cauchy sequence in $M+N$. Each $f_{n}$ decomposes uniquely as $f_{n}=v_{n}+w_{n}+g_{n}$ with $v_{n}$ in $V$, $w_{n}$ in $W$, and $g_{n}$ in $M \cap N$. Since $V+W \perp M \cap N$, we have $\left\|f_{n}-f_{m}\right\|^{2}=\left\|\left(v_{n}-v_{m}\right)+\left(w_{n}-w_{m}\right)\right\|^{2}+\| g_{n}$ $-g_{m} \|^{2}$. If we assume (iv), then

$$
\left\|f_{n}-f_{m}\right\|^{2} \geqslant\left\|g_{n}-g_{m}\right\|^{2}+(1-c)\left(\left\|v_{n}-v_{m}\right\|^{2}+\left\|w_{n}-w_{m}\right\|^{2}\right) .
$$

So $\left(v_{n}\right),\left(w_{n}\right)$, and $\left(g_{n}\right)$ must converge to $v, w$, and $g$ respectively in the closed spaces $V, W$, and $M \cap N$. So $f_{n} \rightarrow v+w+g$. This is in $M+N$, so $M+N$ is closed, and (iv) implies (i). We have (i), (ii), (iii), and (iv) are equivalent. The norm conditions (v) through (viii) are readily shown to be equivalent to condition (iii).

LEMMA 3.2. If the equivalent conditions of Lemma 3.1 hold, then $P+Q$ is 
bounded below on $M+N$. More precisely, for $f$ in $M+N,\|(P+Q) f\|^{2} \geqslant((1$ $\left.-c)^{3} / 2(1+c)\right)\|f\|^{2}$.

Proof. Decompose $f$ as $f=g+v+w$ with $g$ in $M \cap N$, $v$ in $V$, and $w$ in $W$. Then

$$
\begin{aligned}
\|(P+Q) f\|^{2} & =4\|g\|^{2}+\|P(v+w)+Q(v+w)\|^{2} \\
& \geqslant 4\|g\|^{2}+(1-c)\left(\|v+P w\|^{2}+\|w+Q v\|^{2}\right) \\
& \geqslant 4\|g\|^{2}+(1-c)\left((\|v\|-\|P w\|)^{2}+(\|w\|-\|Q v\|)^{2}\right) .
\end{aligned}
$$

If $\|v\| \geqslant\|w\|$, then

$$
\begin{aligned}
\|(P+Q) f\|^{2} & \geqslant 4\|g\|^{2}+(1-c)(\|v\|-\|P w\|)^{2} \\
& \geqslant 4\|g\|^{2}+(1-c)(\|v\|-c\|w\|)^{2} \\
& \geqslant 4\|g\|^{2}+\left((1-c)^{3} / 2\right)\left(\|v\|^{2}+\|w\|^{2}\right) .
\end{aligned}
$$

If $\|w\| \geqslant\|v\|$, apply a similar argument to the term $(\|w\|-\|Q v\|)^{2}$ to obtain the same inequality. In either case,

$$
\begin{aligned}
\|(P+Q) f\|^{2} & \geqslant\|g\|^{2}+\left((1-c)^{3} / 2\right)\left(\|v\|^{2}+\|w\|^{2}\right) \\
& \geqslant\|g\|^{2}+\left((1-c)^{3} / 2(1+c)\right)\|v+w\|^{2} \\
& \geqslant\left((1-c)^{3} / 2(1+c)\right)\left(\|g\|^{2}+\|v+w\|^{2}\right) \\
& \geqslant\left((1-c)^{3} / 2(1+c)\right)\|f\|^{2} .
\end{aligned}
$$

The next lemma allows us to go from spans to intersections.

Lemma 3.3. If $M+N$ is closed, then $M^{\perp}+N^{\perp}$ is closed.

Proof. We have $\left(M^{\perp} \cap N^{\perp}\right)^{\perp}=M+N$ since $M+N$ is closed. Suppose $f$ is in $M+N$ and $f=g+v+w$ as before. Then

$$
\begin{aligned}
\|(1-P)(1-Q) f\|^{2} & =\|(1-P)(1-Q) v\|^{2}=\|(1-Q) v\|^{2}-\|P(1-Q) v\|^{2} \\
& =\|(1-Q) v\|^{2}-\|(P+Q)(1-Q) v\|^{2} \\
& \leqslant\left(1-(1-c)^{3} / 2(1+c)\right)\|(1-Q) v\|^{2}
\end{aligned}
$$

by Lemma 3.2. But $Q v$ is the best approximation to $v$ in $N$, so

$$
\begin{aligned}
\|(1-P)(1-Q) f\|^{2} & \leqslant\left(1-(1-c)^{3} / 2(1+c)\right)\|v+w\|^{2} \\
& \leqslant\left(1-(1-c)^{3} / 2(1+c)\right)\|f\|^{2} .
\end{aligned}
$$

So the restriction of $(1-P)(1-Q)$ to $\left(M^{\perp} \cap N^{\perp}\right)^{\perp}$ has norm strictly less than 1. Lemma 3.1 shows $M^{\perp}+N^{\perp}$ is closed.

4. Proofs of 1.1 and 1.2. Let $P$ and $Q$ be as before, and let $R$ be the projection onto $M+N$. Let $D=P+Q+1-R$. The operator $P \rightarrow Q$ is 0 
on $(M+N)^{\perp}$ and is bounded below on $M+N$ by Lemma 3.2. So its range, which is the same as that of its restriction to $M+N$, is closed. We have

$(P+Q) H=\left(\operatorname{ker}(P+Q)^{*}\right)^{\perp}=(\operatorname{ker}(P+Q))^{\perp}=(M+N)^{\perp \perp}=M+N$.

So $P+Q$ is an invertible map of $M+N$ onto $M+N$ and is 0 on $(M+$ $N)^{\perp}$. Thus $D$ is invertible and we have $D R=R D=P+Q$. Suppose $M$ and $N$ are essentially invariant for $T$. Then $T P-P T P$ and $T Q-Q T Q$ are compact. So

$$
(1-R)((T P-P T P)+(T Q-Q T Q)) D^{-1}=T R-R T R
$$

is also compact, and $M+N$ is essentially invariant. Also, $M^{\perp}$ and $N^{\perp}$ are essentially invariant for $T^{*}$. By Lemma 3.3, $M^{\perp}+N^{\perp}$ is closed, so by the above argument, $M^{\perp}+N^{\perp}$ is essentially invariant for $T^{*}$. Thus $M \cap N=$ $\left(M^{\perp}+N^{\perp}\right)^{\perp}$ is essentially invariant for $T$. If $M$ and $N$ are essentially reducing for $T$, they are essentially invariant for both $T$ and $T^{*}$. By the above, $M+N$ and $M \cap N$ are essentially invariant for both $T$ and $T^{*}$, so they essentially reduce $T$. This completes the proof of Proposition 1.1.

To prove Proposition 1.2, we assume $P Q \in K(H)$. Since $M \cap N$ and $U=(M \cap N)^{\perp}$ reduce $P, Q$, and anything in the $C^{*}$-algebra they generate, we have $\left\|\left.P Q\right|_{U}\right\|^{2}=\left\|\left(\left.P Q\right|_{U}\right)^{*}\left(\left.P Q\right|_{U}\right)\right\|=\left\|\left.Q P Q\right|_{U}\right\|$. This last is a positive, compact, selfadjoint operator of norm no more than 1 . By the spectral theory for compact operators, the norm is the same as the largest eigenvalue. But if $Q P Q f=f$, then $f=Q^{2} P Q f=Q f$ so that $f$ is in $N$. Also, $\|(1-P) f\|^{2}=\langle(1-P) f, f\rangle=\langle(1-P) Q f, Q f\rangle=\langle Q f-Q P Q f, f\rangle=0$. So $(1-P) f=0$, and $f=P f \in M$. Thus $f \in M \cap N$. So the largest eigenvalue of $\left.Q P Q\right|_{U}$, and so also its norm, must be strictly less than 1. Therefore $\left\|\left.P Q\right|_{U}\right\| \subsetneq 1$, and $M+N$ is closed by Lemma 3.1.

5. Remarks. After this paper was completed, the author learned of the following related result of Simon [6, Theorem A.1].

Proposition 5.1. Let $P$ and $Q$ be projections onto subspaces $M$ and $N$ and suppose $P Q$ is compact. Let $R$ be the projection onto the (closed) subspace $M+N$. Then $P+Q-R$ is compact.

Proof. As in the proof of Proposition 1.1, $D=P+Q+1-R$ is invertible. Since $(P+Q-R) D=P Q+Q P$ is compact, the result follows. As in [6], this extends to sums of a finite number of almost (essentially) mutually orthogonal projections by induction.

J. P. Williams was kind enough to suggest this alternative proof of Proposition 1.1. The key step above was to show $P+Q=R D$ for invertible $D$. Corollary 1 of Theorem 2.1 of [5] and the remarks following it show that this follows if $(P+Q) H=R H$. The positive operator $P+Q$ has closed range if and only if its square root does, and in this case,

$$
(P+Q) H=(P+Q)^{1 / 2} H \text {. }
$$


But by Crimmins' formula, Theorem 2.2 of [5], we have

$$
(P+Q)^{1 / 2} H=\left(P P^{*}+Q Q^{*}\right)^{1 / 2} H=P H+Q H=M+N .
$$

This is closed by hypothesis and is equal to $R H$, so the result follows.

Finally, if $J$ is any two-sided ${ }^{*}$-ideal in $L(H)$, we can define $J$-invariant and $J$-reducing subspaces modulo $J$ in the obvious way. The above arguments show that if $M+N$ is closed, then $M \cap N$ and $M+N$ are $J$ invariant or $J$-reducing whenever $M$ and $N$ are.

The author would like to thank Professor Donald Sarason for several helpful suggestions.

\section{REFERENCES}

1. W. B. Arveson, Notes on extensions of $C^{*}$-algebras, Duke Math. J. 44 (1977), 329-355.

2. J. W. Calkin, Two-sided ideals and congruences in the ring of bounded operators in Hilbert space, Ann. of Math. 42 (1941), 839-873.

3. J. Dixmier, Etude sur les variétés et les opérateurs de Julia, Bull. Soc. Math. France 77 (1949), 11-101.

4. P. A. Fillmore, J. G. Stampfli and J. P. Williams, On the essential numerical range, the essential spectrum, and a problem of Halmos, Acta Sci. Math. (Szeged) 33 (1972), 179-192.

5. P. A. Fillmore and J. P. Williams, On operator ranges, Advances in Math. 7 (1971), $254-281$.

6. B. Simon, Geometric methods in multiparticle quantum systems, Comm. Math. Phys. 55 (1977), 259-274.

7. D. Voiculescu, A non-commutative Weyl-von Neumann theorem, Rev. Roumaine Math. Pures Appl. 21 (1976), 97-113.

Department of Mathematics, University of California-Berkeley, Berkeley, California 94720 